\title{
Extended version of the Lee Silverman Voice Treatment (LSVT) results in changes to vocal sound pressure level comparable to those resulting from traditional LSVT in a group of 12 participants ${ }^{1}$
}

Megan J. McAuliffe (Commentary author), Department of Communication Disorders, University of Canterbury, New Zealand

(1) Does an extended version of the Lee Silverman Voice Treatment (LSVT), completed over a 2-month period, result in significant increases in vocal sound pressure level in patients with Parkinson's disease? (2) If so, are these increases comparable to those in a previous LSVT study? (3) Does this extended version of LSVT result in functional improvement to communication?

\section{METHODS}

Design: A pre-test-post-test design was completed in order to determine if an extended Lee Silverman Voice Treatment (LSVT) program (LSVT-X) results in significant increases to vocal sound pressure level (SPL) and significant functional improvement in communication. LSVT-X results for vocal SPL and a perceptual rating of speech were then compared with the results of a previous study examining traditional LSVT (Ramig, Sapir, Fox, \& Countryman, 2001), to determine whether any changes observed were comparable to previous LSVT findings.

Allocation: As the study involved a single LSVT-X group, allocation was not required. However, deception was employed, with the authors informing participants that they would be allocated to either a 1 -month or 2-month intervention group. The authors stated that this was undertaken to ensure participant motivation levels were consistent with previous studies.

Blinding: Unblinded. As no specific information was provided, it was assumed that blinding was not conducted. The authors stated that data collection was not conducted by the treating therapist; however, it would seem that both the treating therapist and assessor were aware of the purpose of the study. In addition, no information was provided regarding blinding of those who conducted the perceptual ratings; therefore, it is assumed that they too were aware of the study purpose.

Study length: The study was conducted over a 6-month period.

Setting: The setting was not specified. It was probably conducted at the National Center for Voice and Speech, in Denver, Colorado, USA.

'Abstracted from: Spielman, J., Ramig, LO Mahler, L, Halpern, A., \& Gavin, W.J. (2007). Effects of an extended version of the Lee Silverman Voice Treatment on voice and speech in Parkinson's disease. American Journal of Speech-Language Pathology, 16, 95-107.

For correspondence: E-mail: megan.mcauliffe@canterbury.ac.nz

Source of funding: Study supported by R01 DC00150 National Institutes of Health.
28 Participants: 12 individuals (9 male, 3 female) with idiopathic Parkinson's disease were included in the study. All participants exhibited speech characteristics typical of Parkinson's disease, were on stable medical regimens during the study, lived independently, and exhibited cognitive abilities adequate to complete all assessment and treatment tasks. Mean age of the group was $67.2 \pm 10$ years, with an average disease duration of $4.8 \pm 3.1$ years.

R Intervention: Participants completed an extended version of the traditional LSVT program. This was named LSVT-X for the purposes of the study. Traditional LSVT is conducted in 1-h treatment sessions, four times per week for four consecutive weeks (a total of 16 1-h sessions over a 4-week period). For full details of LSVT treatment tasks and treatment hierarchy see Ramig, Countryman, O'Brien, Hoehn, and Thompson (1996). In contrast to traditional LSVT, LSVT-X comprised two 1-h sessions per week for eight consecutive weeks. Treatment tasks followed the same hierarchy as traditional LSVT treatment; however, they were spread over a 2-week, rather than 1-week, period. Homework tasks were conducted as per traditional LSVT, that is, 5-10 min on treatment days and 20-30 min on non-treatment days. Therefore, given the extended duration of LSVT-X, participants in this study undertook significantly more home practice than those who have previously completed traditional LSVT. Specific measures of treatment fidelity were not included. It was noted that the treating therapist was a certified LSVT practitioner.

Outcomes: Objective outcome measures included vocal SPL in sustained phonation, reading, picture description and conversational speech. Functional improvement was assessed using the Voice Handicap Index (VHI) (Jacobson et al., 1997), a questionnaire that examines psychosocial function related to voice. Speech outcomes were rated on a visual analogue scale. Specifically, four speech pathology graduate students listened to sentence pairs (one sentence collected pre-treatment, the other post-treatment, taken from the Rainbow Passage) and rated whether the second sample sounded 'better' or 'worse' than the previous sample. Anchor points were -50 (much worse) and +50 (much better). A rating of 0 indicated 


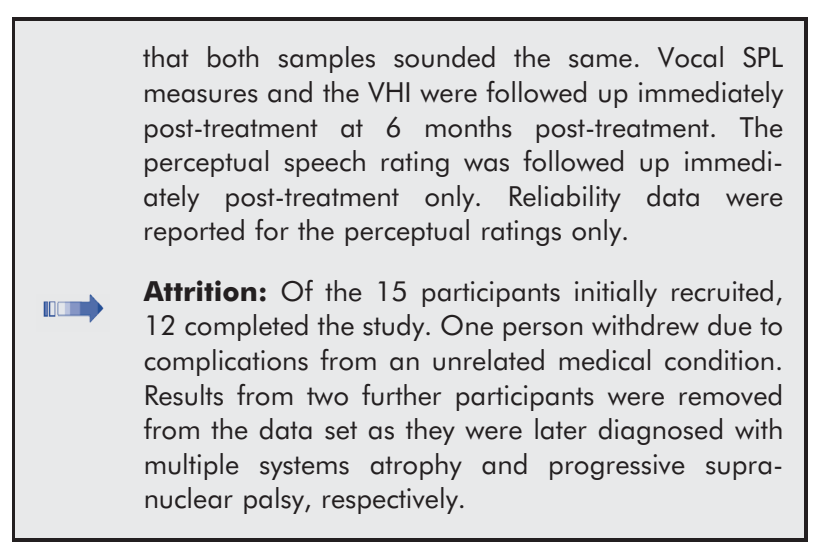

\section{MAIN RESULTS}

LSVT-X resulted in significant improvements to vocal SPL across all conditions (sustained phonation, reading, picture description, and conversation) immediately following treatment. Significant increases in vocal SLP were also observed from pretreatment to 6-month follow-up for sustained phonation, reading, and picture description. Although vocal SPL in conversation increased between the pre-treatment and 6-month followups, this difference was not statistically significant. Across all conditions, there were no significant decreases in vocal SPL between the post-treatment and 6-month follow-ups.

When the results of LSVT-X group were compared with the group results of those who received traditional LSVT in a previous study (Ramig et al., 2001), the groups exhibited similar pre-treatment vocal SPL across all conditions. In addition, the

35 groups exhibited similar vocal SPL levels at the post-treatment and 6-month follow-ups. Only one significant difference was observed, with vocal SPL for the picture description task significantly increased post-treatment in the LSVT-X group.

40 As expected, the LSVT-X group exhibited significantly higher vocal SPL at post-treatment and 6-month follow-ups when compared with the group from Ramig et al. (2001), which received no treatment.

Functionally, there was no significant change in VHI scores for the LSVT-X group post-treatment. When individual scores were examined, 33\% of participants reported significant improvements to voice handicap following treatment. None of the

50 participants exhibited significant worsening immediately after or at 6 months after treatment. The perceptual speech ratings of the LSVT-X group in the current study and traditional-LSVT and no-treatment groups in Ramig et al.'s study (2001)

55 were compared. The LSVT-X and LSVT groups' speech was rated significantly 'better' immediately post-treatment, compared with the group who did not receive treatment.

\section{AUTHORS' CONCLUSIONS}

The group of 12 participants who received LSVT-X exhibited significantly increased vocal SPL following treatment. In addition, their speech was perceived as 'better' immediately post-treatment. These findings appeared consistent with the results of traditional LSVT collected from a previous study (Ramig et al., 2001). Overall, self-ratings on the VHI suggested that participants were less negatively affected by their voices following LSVT-X.

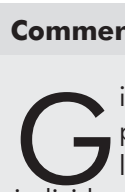
literature in the area of speech intervention for individuals with Parkinson's disease. The findings of this study are promising. However, as acknowledged by the authors, further research including larger participant numbers, and prospective control groups (e.g. traditional LSVT and no treatment) is required to determine the efficacy of LSVT-X as a model of service delivery for people with Parkinson's disease. One particular limitation of this study was the lack of blinding. The article stated that "no treating therapist collected data, and therapists were kept out of sight during data collection to avoid acting as external cues or biasing data collection" (Spielman, Ramig, Mahler, Halpern, \& Gavin, 2007, p. 98). It is therefore assumed that those assessing and treating participants were aware of the study purposes. As stated in the text, "participants were never cued for vocal loudness during data collection sessions" (Spielman et al., 2007, p. 98). However, inadvertent cueing can introduce unavoidable bias into the study results. Future LSVT-X studies would be strengthened by the use of blinding, particularly blinding of assessors. In addition, the inclusion of specific measures of treatment fidelity would further strengthen the study design.

The perceptual assessment of speech could also be strengthened for future studies. Firstly, it is unknown why the authors chose to undertake speech analysis for the pretreatment and immediately post-treatment conditions only. The required stimuli were collected at 6 months in both the present study and Ramig et al. (2001). It would have been of interest to readers to know whether speech improvements were maintained 6 months following treatment. Secondly, the number of listeners included (four) was quite small for a perceptual speech analysis. Thirdly, it is assumed that listeners were not blinded to the study purpose, which introduces the possibility of bias. Given the small number of listeners, lack of follow-up, and potential for bias, only limited conclusions can be drawn from the speech analysis.

Regarding the $\mathrm{VHI}$ results, overall, the average self-rating score dropped from 44 to 30 immediately post-treatment, and rose to 32 at 6 -month follow up. The drop immediately posttreatment was interpreted as a drop from intermediate to mild voice handicap. This change was not statistically significant, which might be related to the small number of participant. authors noted that four participants exhibited a significant improvement in voice handicap immediately post-treatment. 
This was maintained by three participants at 6 months posttreatment. Without seeing the full data set, it is difficult to comment; however, the authors conclusions that "self-ratings using the VHI also suggest that, on the whole, participants who received LSVT-X were less negatively affected by their voices following treatment and did not perceive decline over a 6-month period" (Spielman et al., 2007, p. 103) seems a slightly generous interpretation of the current findings.

In summary, this study provides some evidence that LSVT-X results in similar improvements to speech and voice as does traditional LSVT. However, the comparisons to traditional LSVT are made across studies, rather than within this study, and thus must be viewed as preliminary. Additionally, the results of the study should be interpreted with caution given the small number of participants and lack of control groups. However, the potential for modified treatment schedules is a promising line of enquiry, and future research will be of great interest to clinicians and researchers in the area of motor-speech disorders.

\section{REFERENCES}

Jacobson, B., Johnson, A., Grywalski, C., Silbergleit, A., Jacobson, G., \& Benninger, M., et al. (1997). The Voice Handicap Index (VHI): Development and validation. American Journal of Speech-Language Pathology, 6, 66-70.

Ramig, L. O., Countryman, S., O’Brien, C., Hoehn, M. M., \& Thompson, L. (1996). Intensive speech treatment for patients with Parkinson's disease: Short and long-term comparison of two techniques. Neurology, 47, 1496-1504.

Ramig, L. O., Sapir, S., Fox, C. M., \& Countryman, S. (2001). Changes in vocal loudness following intensive voice treatment (LSVT) in individuals with Parkinson's disease: A comparison with untreated patients and normal aged-matched controls. Movement Disorders, 16, 79-83.

Spielman, J., Ramig, L. O., Mahler, L., Halpern, A., \& Gavin, W. J. (2007). Effects of an extended version of the Lee Silverman Voice Treatment on voice and speech in Parkinson's disease. American Journal of Speech-Language Pathology, 16, 95-107. 